\title{
Surface Instabilities and Nuclear Multifragmentation
}

\author{
L.G. Mcretto, Kin Tso, N. Colonna and G.J. Wozniak \\ Nuclear Science Division, Lawrence Berkeley Laboratory \\ University of California, Berkeley, California 94720, USA
}

March, 1992

This work was supported by the Director, Office of Energy Research, Division of Nuclear Physics of the Office of High Energy and Nuclear Physics of the U.S. Department of Energy under Contract DE-AC03-76SF00098 


\title{
Surface instabilities and nuclear multifragmentation
}

\author{
L.G. Moretto, Kin Tso, N. Colonna and G. J. Wozniak \\ Nuclear Science Division, Lawrence Berkeley Laboratory, 1 Cyclotron Road, \\ Berkeley, CA 94720
}

\begin{abstract}
Central heavy-ion collisions, as described by a Boltzman-Nordheim-Vlasov calculation, form nuclear disks that break up into several fragments due to surface instabilities of the Rayleigh-Taylor kind. We demonstrate that a sheet of liquid, nuclear or otherwise, stable in the limit of infinitely sharp surfaces, becomes unstable due to surface-surface interactions. The onset of this instability is determined analytically. The retevance of these instabilities to nuclear multifragmentation is discussed.
\end{abstract}

\section{Introduction}

Multifragmentation is undoubtedly the most striking process observed so far in intermediate-energy heavy-ion reactions ${ }^{1-5}$. The models designed to explain it can be divided into statistical and dynamical models 6 . Statistical models range from a standard sequential-statistical-binary-decay model 7 on the one hand, to simultaneous multifragment decay models $8-10$ on the other. Dynamical models are even more varied. They range from nuclear shattering ${ }^{11}$ to spinodal instability ${ }^{12-14}$. The latter is associated with the transit of a homogeneous fluid across a domain of negative pressure, which leads to its breaking up into droplets of denser liquid embedded in a lower density vapor. Since the spinodal instability can occur in an infinite system, it can be called a bulk or volume instability. Percolation models have also been used to describe this liquid-vapor transition 15 .

\section{Surface Instabilities in Boltzmann-Nordheim-Vlasov Calculations}

Here we want to consider another class of instabilities that may play an important, if not a dominant role in multifragmentation, namely surface instabilities of the Rayleigh-Taylor kind16. These instabilities are called surface instabilities because they depend on the presence of a surface endowed with surface tension.

Our attention to these instabilities was drawn by some peculiar results of numerically simulated heavy-ion collisions. We simulated head-on collisions of two nearly symmetric heavy-ions using the Boltzmann-Nordheim-Vlasov (BNV) equation (which contains both mean field and collision terms) within a test particle approach in a full ensemble method 17-19, with each nucleon being represented by 40 test particles 20 . During these calculations, we noticed two interesting features. First, during the collision process a "disk" develops due to the side-squeezing of nuclear matter, whose thickness decreases and diameter increases monotonically with increasing bombarding energy. Second, if the disk becomes sufficiently thin, it 
breaks up into several fragments of a size commensurate with the thickness of the disk.

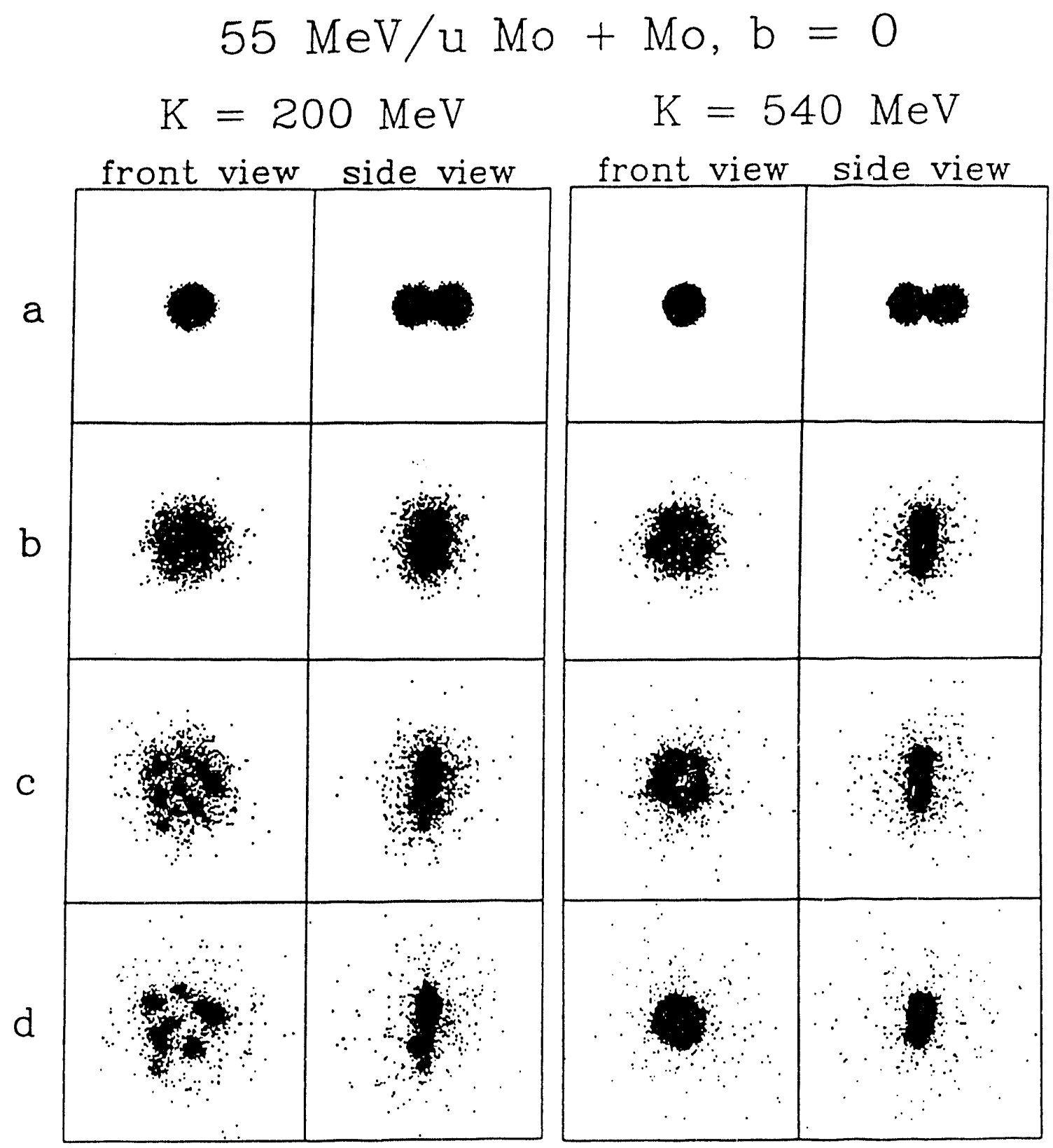

Figure 1. BNV calculations for a head-on collision $(b=0)$ of the $55 \mathrm{MeV} / \mathrm{u} 90 \mathrm{Mo}+90 \mathrm{Mo}$ reaction at time steps of (a) 20, (b) 60 , (c) 120 , and (d) $180 \mathrm{fm} / \mathrm{c}$. The front and side-views of the colliding systems are given in columns $1 \& 2$, respectively for a value of the incompressibility constant, $\mathrm{K}=200 \mathrm{MeV}$. Similar views are shown in columns $3 \& 4$ for $\mathrm{K}=540 \mathrm{MeV}$. 
Some of these features are shown in Figs. 1-3 for head-on collisions of two $90 \mathrm{Mo}$ nuclei at three bombarding energies and two extreme values of the incompressibility constant $\mathrm{K}$. In these figures, the front and side-views of the colliding systems are shown in the rows $(a-d)$ corresponding to four different times: $t=20,60,120$, and $180 \mathrm{fm} / \mathrm{c}$, respectively.

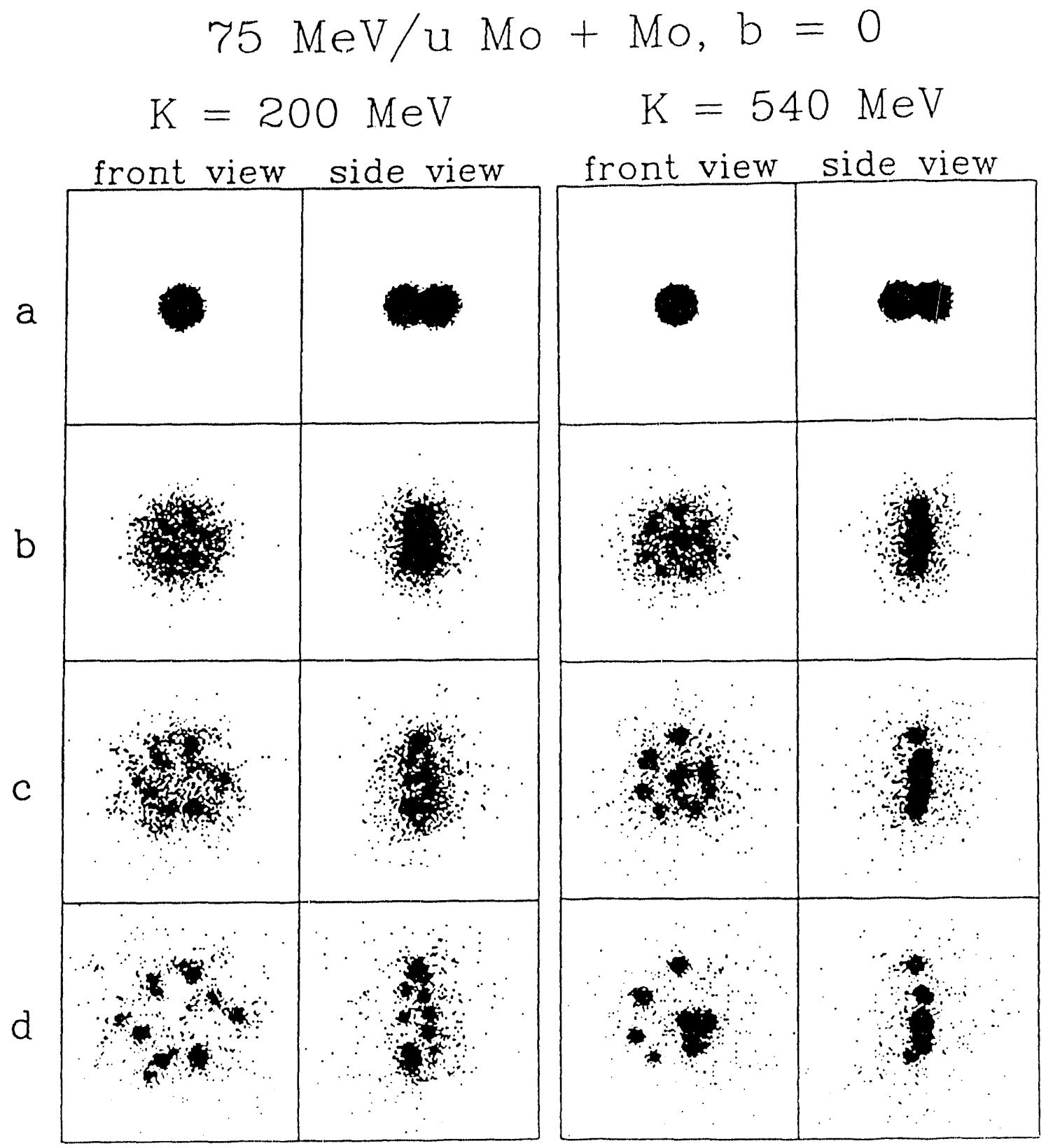

Figure 2. Same as Fig. 1 for the $75 \mathrm{MeV} / \mathrm{u} 90 \mathrm{Mo}+30 \mathrm{Mo}$. 
For $\mathrm{K}=540 \mathrm{MeV}$ and the lowest bombarding energy, a thick disk forms and some mottling develops at its maximum extension (incipient fragment formation). However, the mottling heals and the disk falls back to a more or less spherical blob. At higher bombarding energy, the disk becomes thinner, with a larger diameter than in the previous case. As the collision progresses, the mottling appears and develops rapidly into a beautiful crown of many fragments of approximately the same size, that slowly separate due to the residual kinetic energy of the disk and their mutual Coulomb repulsion. In some cases, two or more of these protofragments coalesce into a larger fragment (see for example Fig. 2, column 3).

$$
\begin{gathered}
100 \mathrm{MeV} / \mathrm{u} \mathrm{Mo}+\mathrm{Mo}, \mathrm{b}=0 \\
\mathrm{~K}=200 \mathrm{MeV} \quad \mathrm{K}=540 \mathrm{MeV}
\end{gathered}
$$

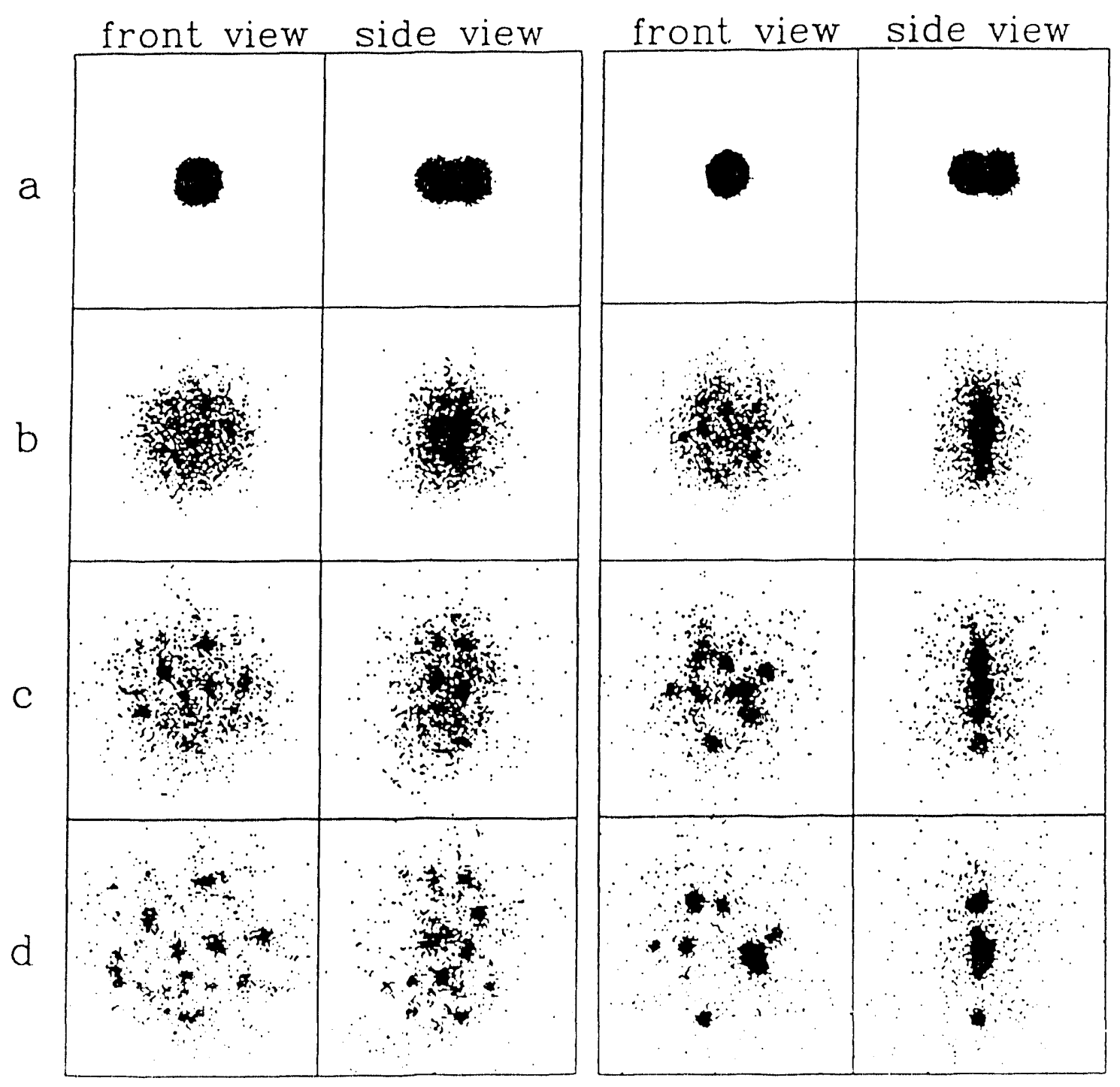

Figure 3. Same as Fig. 1 for the $100 \mathrm{MeV} / \mathrm{u} 90 \mathrm{Mo}+90 \mathrm{Mo}$. 
We repeated the calculations for $K=200 \mathrm{MeV}$ in order to cover the range of nuclear incompressibility currently believed appropriate for nuclear matter. At 55 $\mathrm{MeV} / \mathrm{u}$ and $\mathrm{K}=200 \mathrm{MeV}$, a thin disk is formed and fragment formation occurs, in contrast to the high incompressibility case where fragment formation does not occur as yet. At higher bombarding energies, fragment formation is observed for both values of the incompressibility. However, for the high incompressibility cases, the disks are much sharper, and the mottling and fragment formation stand out more clearly. Similar calculations have been performed for a range of central impact parameters and entrance-channel mass asymmetries with similar results.

\section{Metastability of a sheet of liquid}

The overall appearance of the disk fragmentation strongly suggests that it is caused by surface instabilities. More precisely, the system escapes from the high surface energy of the disk by breaking up into a number of spherical fragments with less overall surface. Thus, fragment formation, in this picture depends only on the presence of a surface energy term. (In the static limit, the BNV model becomes equivalent to the Hartree Fock model, which can reproduce the nuclear masses throughout the periodic table and thus expresses a good surface energy). Multinucleon correlations, which are commonly thought to be essential for fragment formation are actually nct necessary beyond their macroscopic manifestation through the surface energy. Incidentally, the very same observation can be made for volume instabilities.

The observed instability maybe akin to the Rayleigh instability 16 of a cylinder of liquid. The cylinder is unstable with respect to small perturbations of wave length $\lambda$ $\geqq 2 \pi R$, where $R$ is the radius of the cylinder. But, is a disk of liquid, or more gerierally, a sheet of liquid truly unstable?

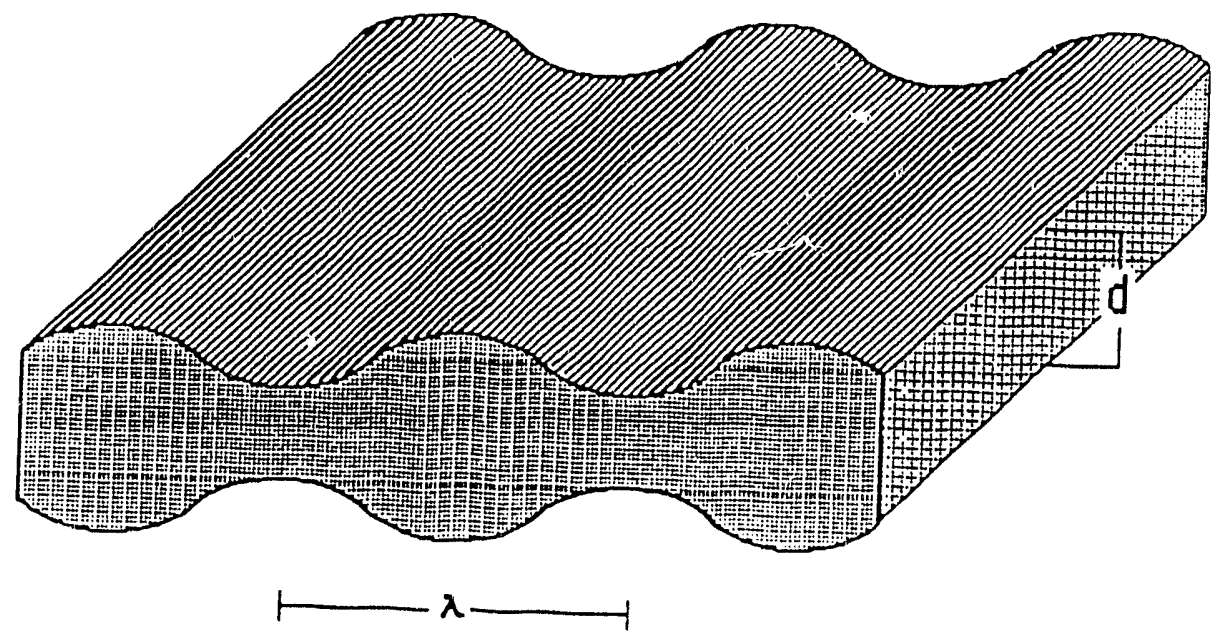

Figure 4. Schematic illustration of the perturbation of a thin sheet of liquid. See discussion in text. 
If we assume sharp surfaces (no surface thickness, no surface-surface interaction), a sheet can be metastable with respect to a break-up into a layer of cylinders or spheres (see Fig. 4). The onset of metastability for both cases is easily calculated. On a sheet of thickness $d$ let us identify stripes of width $\lambda$. These stripes can favorably collapse into cylinders when the surface area of a stripe (top + bottom) is greater than the surface area of the cylinder of equivalent volume. This can be easily shown to occur for

$\lambda \geqq \pi \mathrm{d}$

Similarly, if the sheet is tiled with squares of side $\lambda$, the squares can favorably collapse into spheres when

$\lambda \geqq 3 / 2(2 \pi)^{1 / 2} d$.

These conditions refer to metastability and not necessarily to instability, since there may $k a$ a barrier that prevents the sheet from reaching the more stable configurations illustrated above, and indeed there is. A sheet with sharp surfaces is stable to small perturbations of all finite wavelengths and becomes indifferent to perturbations of infinite wavelengths. Clearly, any wave of infinitesimal amplitude $A$ increases the surface area of the sheet, independent of the sheet thickness, since, in the limit of infinitely sharp surfaces, the surfaces do not know of each other, until they touch (see Fig. 4). The dimensionless surface energy increase can be trivially shown to be:

$\Delta V_{S} \approx \frac{2 \pi^{2}}{\lambda^{2}} A^{2}+$ higher order terms,

where $\lambda$ is the wavelength of the perturbation.

On the other hand, the systems portrayed in Figs. 1-3 develop what appears to be a genuine instability! Perhaps, the system, which has plenty of energy, simply jumps the barrier. But, there is another, more likely possibility.

\section{Instability of a sheet of liquid and surface-surface interactions}

Nuclear surfaces are not sharp, but diffuse, and they interact with each other through an interaction of finite range called also the proximity force $21, \Phi(s)$, where $s$ is the distance between surfaces. We can now calculate the incremental energy of a sheet subjected to a perturbation of wavelength $\lambda$ and small amplitude $A$. The dimensionless proximity interaction is:

$V_{P}=\frac{2}{\lambda} \int_{0}^{\lambda} \Phi(s) d x-\frac{2}{\lambda}\left(P(\lambda)+Q(\lambda) A^{2}\right)$

where

$P(x)=\int_{0}^{\lambda} \Phi_{0}(x) d x$ and $Q(\lambda)=\int_{0}^{\lambda} \Phi_{2}(x) d x$ 
with $s=d+2 A$ sin $k x, \Phi_{0}$ and $\Phi_{2}$ being the zeroth and second order coefficients of the Taylor expansions of $\Phi(A, x)$ about $A=0$, and $k=2 \pi / \lambda$.

The overall energy increase, including the term in Eq. 3, is:

$\Delta V=A^{2}\left(\frac{2 \pi^{2}}{\lambda^{2}}+\frac{Q(\lambda)}{\lambda}\right)$

Instability occurs when the coefficient of $A^{2}$ is zero or negative. Thus, the critical wavelength for the onset of the instability is given by the equation:

$\lambda_{c} Q\left(\lambda_{c}\right)+2 \pi^{2}=0$.

Any perturbation with $\lambda>\lambda_{c}$ is then unstable, namely it will grow spontaneously and indefinitely. Using for the proximity potential the expression in ref. 21 , we obtain

$\lambda_{c}=1.10 \mathrm{~b} \exp [2 \mathrm{~d} / 3 \mathrm{~b}]$

where $b$ is the range of the proximity interaction.

When the thickness of the sheet becomes much greater than the range of the proximity interaction, the critical wavelength tends to infinity. This is the trivial result for infinitely sharp surfaces that was mentioned above. However, when the thickness of the sheet becomes comparable to the proximity range $b$, the critical wave-length decreases very rapidly. This result is quite interesting, because it applies in general to all liquids, and because it is, we believe, new.

\section{Application to nuclear collisions}

How can all this apply to the BNV calculations? The considerations made above are purely static, while the BNV calculations deal with the full dynamical problem. The time aspect, for instance, is reflected in the fact that the thickness of the disk develops in time, though it seems to become nearly stationary as a turning point is reached. In other words, there must be an interplay between the rate of growth of the instabilities and the underlying disk dynamics. Therefore, it may be difficult to interpret the details of these phenomena without incorporating specifically the time evolution of the disk.

In general, it appears that the observed relationship between fragment size and disk thickness is consistent with Eq. 8, if one uses for $b$ the zero temperature value of $\sim 1 \mathrm{fm}$. But Eq. 8 gives only a lower bound for the instability range. It is clear that the disk must become thin enough in order to allow the critical wavelength to fit comfortably within the disk diameter. But, which wavelength, if any, should actually determine the collapse of the disk? This answer cannot be determined from the instability considerations made above. Rather, it depends on how fast the instability develops. Rayleigh showed 16 that, for a cylinder, the instabilities grow exponentially, and that the growth is fastest for $\lambda=9.11 \mathrm{r}$. This result has been obtained assuming irrotational flow and no viscosity. However, it is known that viscosity can play an important role in this respect.

In contrast to an infinitely extended sheet, the finite size of the disk may introduce interesting effects. The nearly symmetric patterns of the fragments 
suggest the presence of stationary waves determined by the boundary conditions of the disk edge. In fact the association of these patterns with the nodal pattern of cylindrical harmonics is very tempting.

We have explored the role of incompressibility in these calculations. The upper value of the incompressibility parameter essentially prevents any compression (and expansion) from occurring. Thus, it should isolate surface effects from those associated with compression and expansion. The overall comparison between the two extreme cases shown in Figs. 2 \& 3 suggests that thinner and sharper disks are formed at high incompressibility. As a matter of fact, at the highest bombarding energy (100 Mev/u) investigated, the low incompressibility calculation shows a coarse fuzzy disk where fragments are seen to form within its thickness in a volume-like process. This may indicate the appearance of a volume (spinodal) instability. It is conceivable that the different geometries for events with low or high incompressibility might be used to obtain an experimental constraint on this important pararneter.

Neck fracture dua Rayleigh instabilities has been postulated in low energy fission 22. We have also explored collisions with much larger impact parameters in search of shapes with long necks that might also be subject to the Rayleigh instabilities. Indeed such necks are observed and the instability is seen to develop.

Concerning the BNV calculations, two apparent puzzles may need clarification:

1) What is the origin of the fluctuations that eventually lead to the observed instabilities?

2) Why certain symmetries contained in the BNV equations (like cylindrical symmetries) are violated when the instabilities manifest themselves?

The ainswer to both points resides in the algorithmic noise (numerical approximations) associated with the solution of the equations. This noise is responsible for both the fluctuations and the breaking of symmetries, which due to the underlying instabilities are inevitably amplified in the evolution of the system.

While algorithmic noise is quite effective in evidentiating instabilities, it is not physical. Therefore, one should not rely on it to generate distributions in fragment number, mass, etc.

\section{Conclusion}

In conclusion, it appears that surface instabilities leading to multifragmentation of disks and necks should be very pervasive in intermediate-energy heavy-ion collisions. Models that contain a surface term in an explicit or implied form are capable of portraying these instabilities, which lead to multifragmentation, without the use of explicit many body correlations.

\section{Acknowledgements}

This work was supported by the Director, Office of Energy Research, Division of Nuclear Physics of the Office of High Energy and Nuclear Physics of the US Department of Energy under contract DE-AC03-76SF00098.
8. References
1. R. Trockel et al., Phys. Rev. C 39 (1989) 729.
2. R. Bougault et al., Phys. Lett. B 232 (1989) 291.
3. Y. D. Kim et al., Phys. Rev. Lett. 63 (1980) 494.
4. Y. Blumenfeld et al., Phys. Rev. Lett. 66 (1991) 576. 
5. C. A. Olgilvie et al., Phys. Rev. Lett. 67 (1991) 1214.

6. For a review on the subject and for relevant references see for instance; $L$. G. Moretto and G. J. Wozniak, Prog. Part. \& Nucl. Phys. 21 (1988) 401.

7. See for instance; R. J. Charity et al., Nucl. Phys. A483 (1987) 371.

8. J. P. Bondorf, R. Donangelo, I. N. Mishustin, C. J. Pethick, H. Schulz, and K. Sneppen, Nucl. Phys. A443 (1985) 321.

9. D. H. E. Gross, L. Satpathy, Ta Chung Meng and M. Satpathy, Z. Phys. A309 (1982) 41.

10. J. Randrup and S. F. Koonin, Nucl. Phys. A356 (1981) 223.

11. J. Aichelin and J. Hüfner, Phys. Lett. 136B (1984) 15.

12. P. J. Siemens, Nature 305 (1983) 410.

13. G. Bertsch and P. J. Siemens, Phys. Lett. 126B (1983) 9.

14. J. A. Lopez and P. J. Siemens, Nucl. Phys. A431 (1984) 728.

15. X. Campi, Phys. Lett. B208 (1988) 351.

16. Lord Rayleigh, article 58, "Scientific Papers", Dover Publication, Inc, New York, p. 361 (1964).

17. A. Bonasera, G. Fu Burgio and M. Di Toro, Phys. Lett. B221 (1989) 233.

18. A. Bonasera, G. Russo and H. H. Wolter, Phys. Lett. B246 (1990) 337.

19. M. Colonna et al., Lawrence Berkeley Laboratory preprint No. LBL-30810.

20. The calculations were done for a range of test particle number. A value of 40 was chosen since the results were stable against a further increase.

21. J. Blocki, J. Randrup, W. J. Swiatecki and C. F. Tsang, Ann. Phys. 105 (1977) 427.

22. U. Brosa, S. Grossmann, A. Müller and E. Becker, Nucl. Phys. A502 (1989) $423 \mathrm{c}$. 

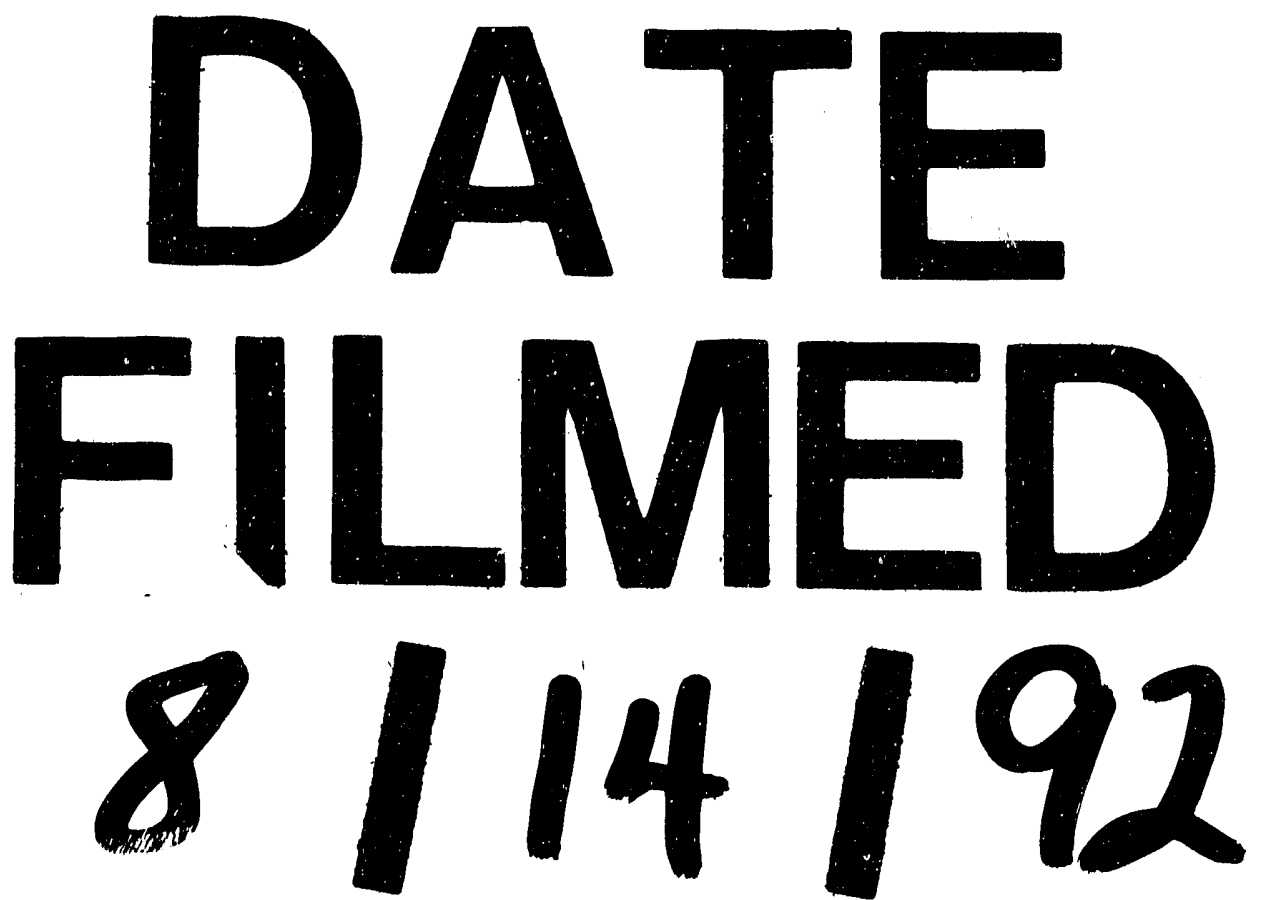
致

,

!

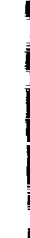

1

I" 